\title{
All-optical signal processing using planar Bragg gratings
}

C. Sima, J. C. Gates, H. L. Rogers, B. D. Snow, C. Holmes, M. N. Zervas, P. G. R. Smith

\author{
Optoelectronics Research Centre, University of Southampton \\ Southampton, United Kingdom \\ cs4g09@orc.soton.ac.uk
}

The fabrication techniques of Bragg gratings broadly fall into two categories: that are holographic, and that are non-interferometric, based on the periodical UV radiation along the photosensitive medium. The fabrication technique in this work is the direct UV grating writing (DGW). This method involves focusing two crossed laser beams $(\lambda=244 \mathrm{~nm})$ into a photosensitive core layer. Precise translation of the sample and modulation of the interference pattern define the channel waveguide and simultaneously create grating structures, shown in Figure 1. First developed at Optoelectronics Research Centre in 2002, it has similarities to the UV writing techniques used for fiber Bragg grating inscription. Advanced grating properties such as chirp, phase shifts, and apodisation are introduced by adjusting the laser intensity and the translating speed.

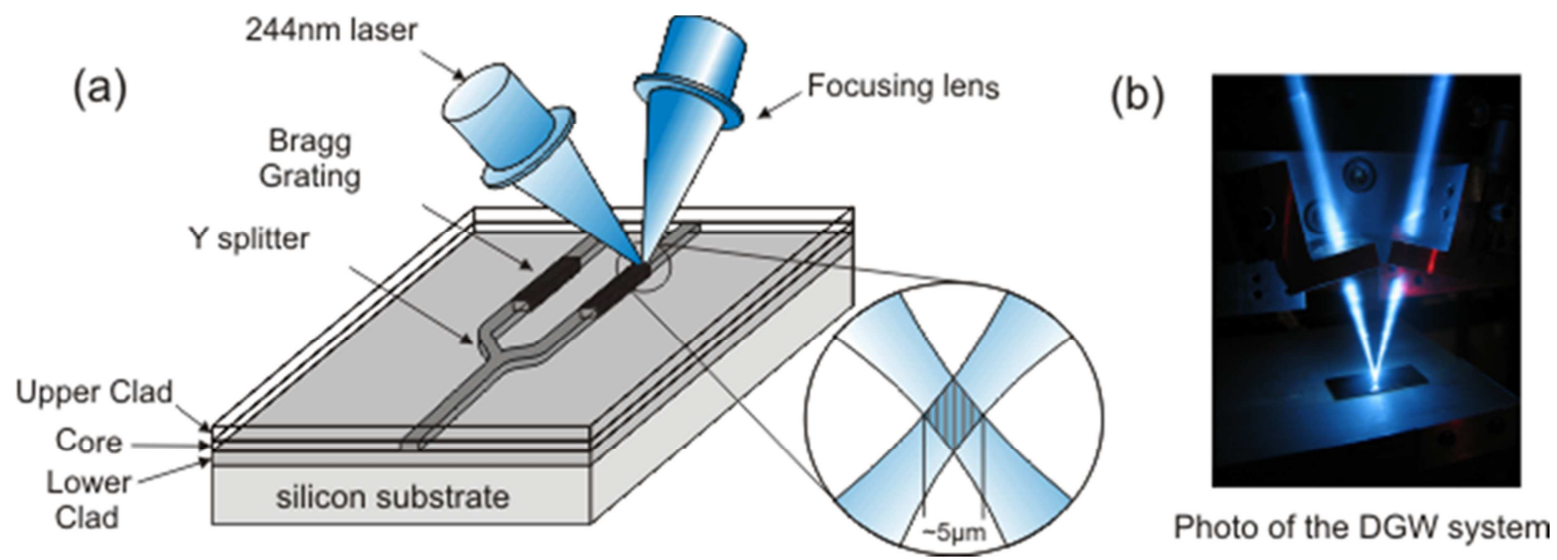

Figure 1 the illustration of (a) direct Grating writing (DGW) setup with two crossed beams focused on the core layer of the silica-on-silicon substrate ; (b) photograph of DGW system.

One significant advantage of the DGW system lies in the small spot size $(<5 \mu \mathrm{m}$ in diameter $)$ of the laser beam, in terms of the width of the interference pattern. Rather than using a wide area exposure that is restricted using a phase mask, DGW uses a focussed optical exposure such that the dimensions of the UV induced structure are determined by the focussed spot size. For complex grating engineering, this feature is superior to the conventional phase mask techniques for accurate control of the chirped, for phase shifts, apodisation and other complex Bragg grating structures. 

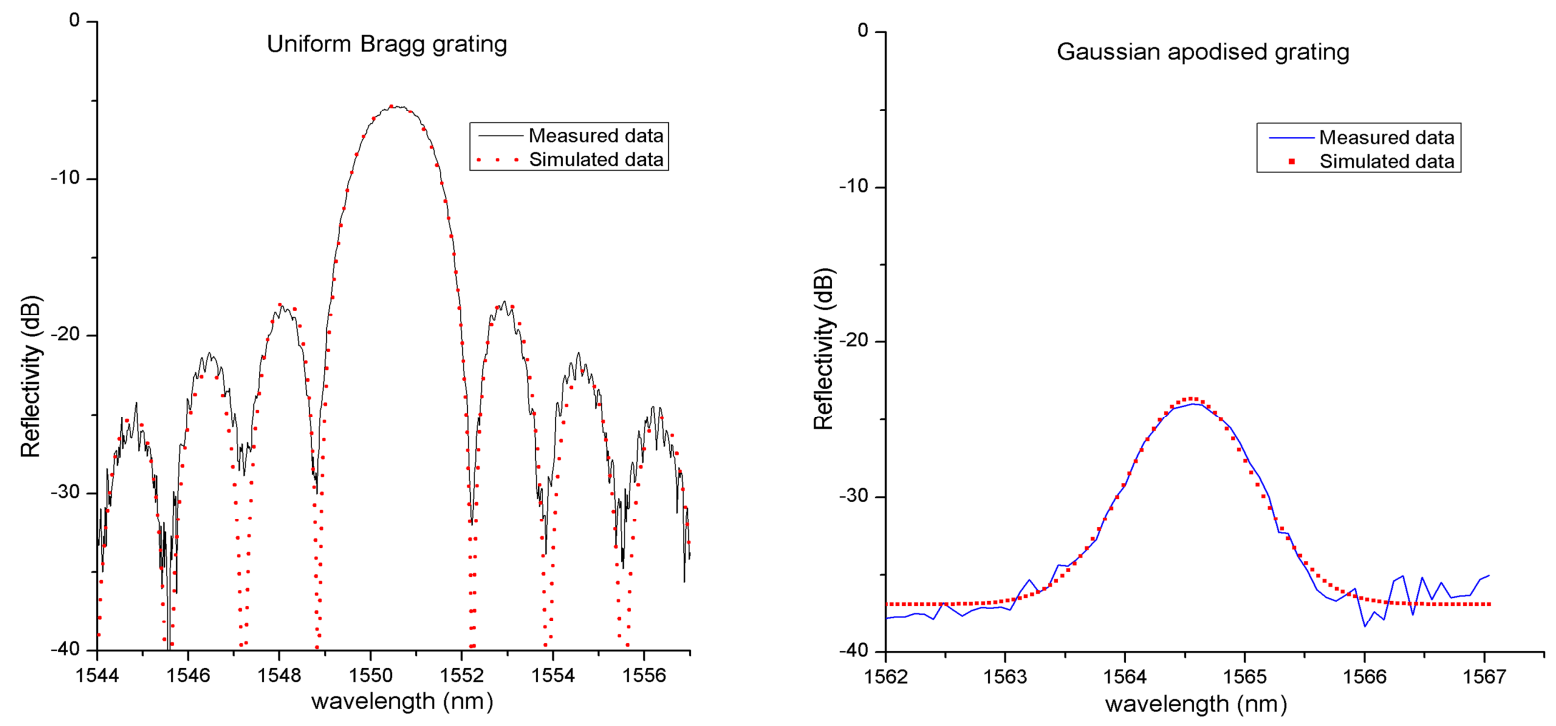

Figure 2 Reflection spectrum of a $0.5 \mathrm{~mm}$ uniform Bragg grating and a $2 \mathrm{~mm}$ Gaussian apodised Bragg grating: experimental results (line) and simulated data (dot).

We will discuss approaches to grating apodisation, for examples, Figure 2, showing Gaussian apodised gratings vs. non-apodised uniform gratings (experimental data and theory modelling results); show how our small writing spot allows fabrication of phase shift devices offering all optical signals processing, such as Figure 3, showing the reflectivity and group delay responses of a $3 \mathrm{~mm} \pi$ phase-shift grating. The small writing spot size also allows fabrication of chirped grating devices, illustrated in Figure 4. The thermal tuning responses of the chirped devices are demonstrated in Figure 5. We will present results on all-optical signal processing using this technique.

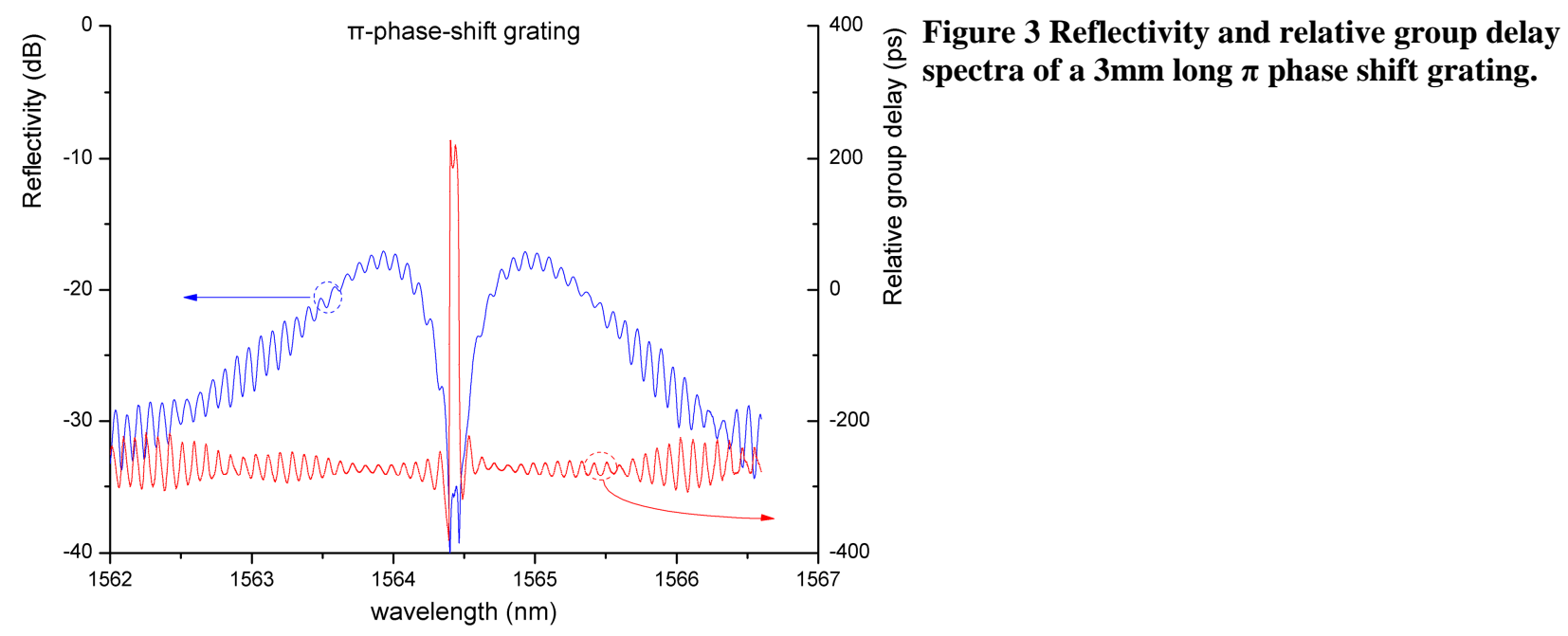



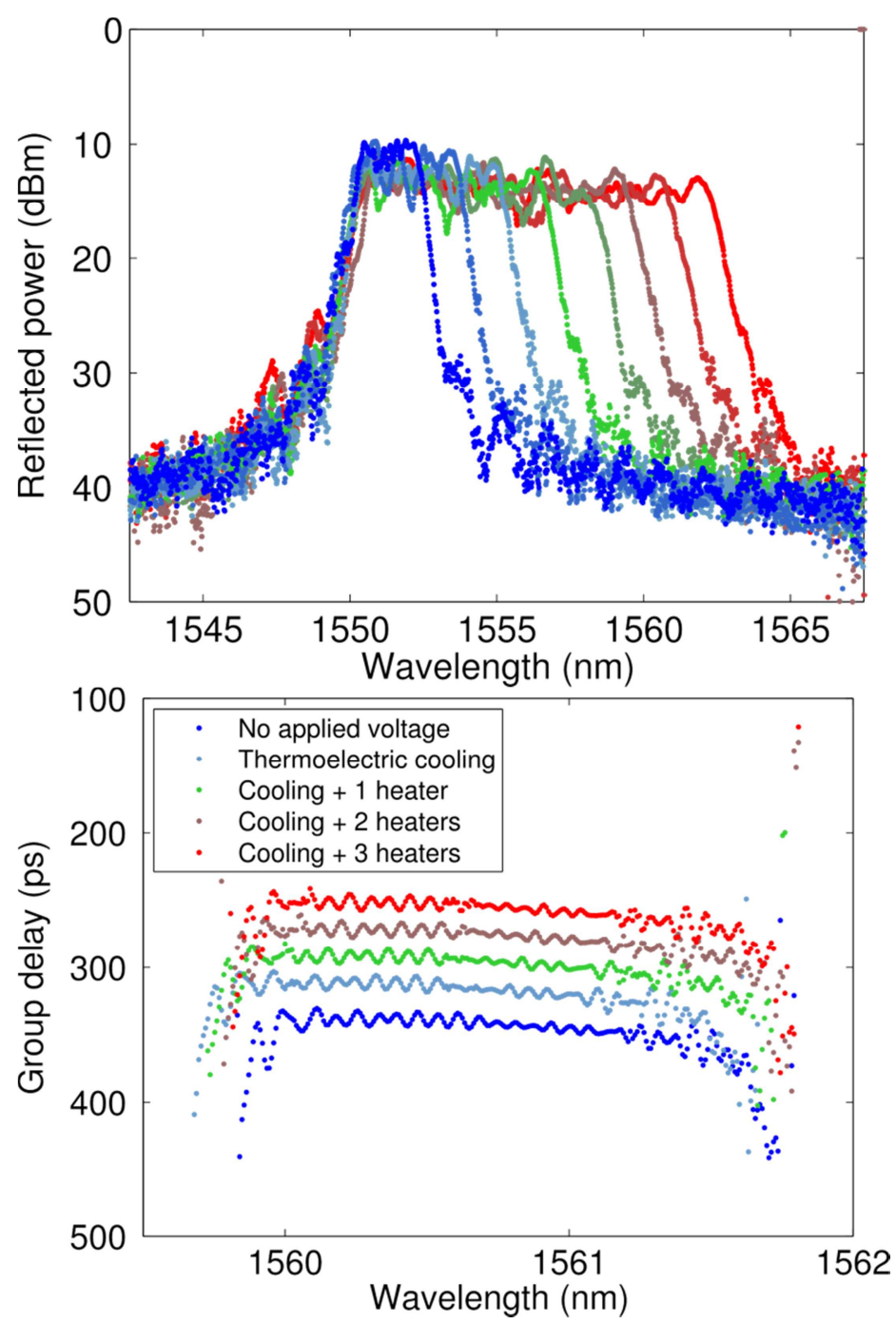

Figure 4 Reflection spectra of $9 \mathrm{~mm}$ long linearly chirped Bragg gratings with chirp rates of 2.8 $14.4 \mathrm{~nm} / \mathrm{cm}$. Data courtesy of Ben Snow.

Figure 5 Group delay spectra of a chirpapodised grating under thermal tuning using a thermoelectric module to cool the silicon base. The spectra are offset for clarity. Data courtesy of Ben Snow. 